\title{
Armaduras de lazo toledanas. Evolución de las trazas geométricas con estrellas de ocho puntas y su relación con los diferentes sistemas constructivos empleados
}

\section{Toledo's wood lattice frames. Eight pointed star geometric patterns'evolution in relationship with the different used constructive systems}

E. Nuere ${ }^{(*)}$, E. Franco ${ }^{(* *)}$, M. C. Fernandez Cabo ${ }^{(* * *)}$

\section{RESUMEN}

La ciudad de Toledo, sede permanente del poder durante casi seis siglos tras la ocupación romana, fue dominada por visigodos y omeyas hasta su conquista castellana. Su evolución arquitectónica la convierte es un enclave idóneo para el desarrollo de la carpintería de armar. Sus armaduras de lazo suponen un magnífico ejemplo donde entender el oficio a través de las posibilidades geométricas y constructivas exploradas por quienes las realizaron. Se analizan las trazas geométricas y su implantación desde los ejemplos más sencillos y numerosos con estrellas de ocho puntas hasta las complejas trazas de geometría radial; la evolución de las armaduras de par y nudillo, que, mediante la prefabricación, posibilitó construir complejas armaduras de siete paños con lacería.

Palabras clave: Lacería, Toledo, mudéjar, armadura, madera, carpintería.

\section{ABSTRACT}

The city of Toledo, permanent seat of power for almost six centuries after the roman occupation, was dominated by visigoths and omeyas until the castilian conquest. Its architectural evolution makes it an ideal place for the development of carpentry. Its interlaced wood structures suppose a magnificent example to understand the relationship between the geometric patterns and the constructive solutions explored by those who made them. Geometrical patterns and how they get to form part of interlaced structures are analyzed from the very simple and most numerous examples with eightpointed stars to the complex patterns of radial geometry. Prefabrication method is studied applied to rafter and collar structures, showing how it will make possible to build complex lattice seven panel structures.

Keywords: Interlace, Toledo, Mudejar, structure, wood, carpentry.

(*) Dr. Arquitecto. Real Academia de Bellas Artes de San Fernando, Madrid (España).

${ }^{(* *)}$ Arquitecto doctorando. Escuela Técnica Superior de Arquitectura. Universidad Politécnica de Madrid (España).

$\left({ }^{* * *}\right)$ Dr. Arquitecto. Escuela Técnica Superior de Arquitectura. Universidad Politécnica de Madrid (España).

Persona de contacto/Corresponding author: elena@loblanco.org (E. Franco)

ORCID: https://orcid.org/oooo-0002-6368-9402 (E. Nuere); https://orcid.org/oooo-0oo2-0520-4585 (E. Franco); https://orcid.org/oooo-0oo2-3393-2774 (M.C. Fernández Cabo)

Cómo citar este artículo/Citation: Nuere, E.; Franco, E.; Fernández Cabo, M. C. (2019). Armaduras de lazo toledanas. Evolución de las trazas geométricas con estrellas de ocho puntas y su relación con los diferentes sistemas constructivos empleados. Informes de la Construcción, 71(556): e317. https://doi.org/10.3989/ic.68659.

Copyright: (C) 2019 CSIC. Este es un artículo de acceso abierto distribuido bajo los términos de la licencia de uso y distribución Creative Commons Reconocimiento 4.0 Internacional (CC BY 4.0). 


\section{ANTEDENTES Y MARCO DE REFERENCIA}

La carpintería de armar española es poco conocida y sólo ha sido parcialmente estudiada a pesar de su abundancia y calidad. El objeto de este artículo es la comprensión formal y constructiva de las armaduras de lazo en la ciudad de Toledo y de la evolución que allí se ha venido desarrollando en la carpintería de armar, habida cuenta de su importancia histórica.

Toledo tiene su origen en una plaza fuerte romana a orillas del Tajo y pasa a convertirse en la ciudad comercial hispano romana de Toletum. Entre el año 507 y el 711 fue capital del Reino Visigodo, Urbs Regia con obispo propio albergando a la población hispano-romana, al oficio palatino, a la comunidad judía y a intelectuales y estudiantes, siendo sede de la importante sucesión de Concilios de Toledo. En el año 711 es conquistada por el Emirato de Córdoba, pasando la nueva Tulaytulah a ser una ciudad importante del emirato e incorporando musulmanes y mozárabes a la población residente. Con la caída del califato, la ciudad se convierte en 1037 en el centro de la Taifa de Toledo. La ciudad es reconquistada en el año 1085, convirtiéndose de nuevo en una ciudad fronteriza a la par que en un importantísimo centro religioso sede del Arzobispado primado de España y de la nueva Catedral, terminando de enriquecer la población ahora formada por el oficio religioso así como por los intelectuales llegados a la Escuela de Traductores de Toledo, a los católicos de rito romano, a mudéjares, judíos y mozárabes. Hay que destacar no sólo la importancia de Toledo sino su temprana reconquista si la comparamos con la de Andalucía: en 1236 la de Córdoba y en 1492 en Granada, lo que sin duda pudo tener su influencia en la carpintería de armar.

El estudio se acota mediante la elección de una muestra formada por las armaduras de lazo ya que éstas reflejan el máximo conocimiento alcanzado tanto en la geometría y trazado como en el oficio de carpintero necesario para su construcción. La elección geográfica de la ciudad de Toledo supone la garantía de que los mejores carpinteros estuvieron allí, sirviendo al poder y materializando sus intereses, fuera el poder hispano romano, visigodo, omeya, bereber o cristiano.

\subsection{Precedentes en el estudio de la carpintería de armar toledana}

El estudio de la carpintería de armar toledana tiene como precedentes los trabajos de Balbina Martínez Caviró, que recopilan armaduras de la ciudad de Toledo y su provincia, las clasifica por tipologías y ofrece referencias iconográficas asociándolas a linajes y fechas y, en ocasiones, a documentación histórica asociada a la construcción de las armaduras (1). En 2007, se publica "Artesonados de Toledo" (2), un trabajo realizado por Enrique Nuere y cuatro profesores de la Escuela de Aparejadores de Madrid, donde los alumnos documentan 71 armaduras Toledanas.

Resulta indispensable en el estudio de Toledo la muy documentada publicación titulada Arquitecturas de Toledo donde se analizan los edificios más significativos desde el punto de vista histórico, arquitectónico y constructivo, apoyándose en planimetrías y situando al estudioso en la complejidad de cada edificio (3).

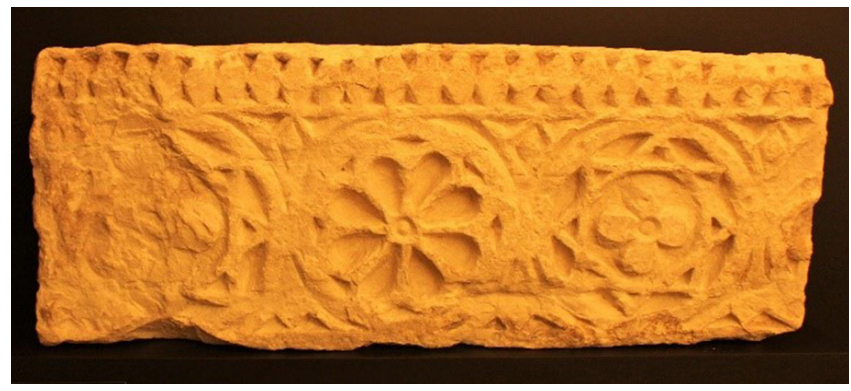

Figura 1. Imposta caliza (siglo VII) y placa de arcilla cocida decorada a molde (época paleocristiana). Museo de los Concilios y la cultura Visigoda. Toledo.

\subsection{La estrella de ocho puntas en Toledo}

El análisis de la carpintería de armar toledana desvela características propias, pero sin duda la más sorprendente de todas ellas, es el uso sistemático y casi exclusivo de la estrella de ocho como base de sus trazados.

La lacería combina la existencia de una cinta que recorre la estructura buscando una continuidad, el lazo, con la estrella. Como cuestión de base cabe preguntarse por los antecedentes del lazo y de la estrella. El lazo se ha usado profusamente por toda la península en los mosaicos romanos, y en la proximidad de Toledo encontramos los de Carranque donde también su composición se desarrolla en torno al octógono; o el Mosaico de los Pescados que se guarda en el Museo de Santa Cruz de Toledo. En cuanto a la estrella de ocho puntas generada a partir de un cuadrado girado sobre sí mismo 45 grados, es en el mismo Toledo donde encontramos ejemplos Visigodos (figura 1).

El mosaico, el barro y la madera permiten dibujar con libertad las bases que posteriormente se tallarán, imprimirán o modelarán. Incluso se puede usar la variante del color y de la textura.

En madera, el ejercicio de composición tiene una constante, la carpintería de armar genera estructuras a partir de troncos de árboles. Ello supone que haya vigas lineales, con unas escuadrías determinadas por las proporciones de la sala. Existe un sistema constructivo que resuelve unas necesidades estructurales, y el diseño debe de ocupar el hueco que deja el cumplimiento de la función estructural. Con un grado de libertad limitado se encuentra un mundo de formas en el que, en el caso de Toledo, la estrella de ocho puntas y el lazo sirven de herramienta compositiva que abarca desde las armaduras más antiguas hasta los artesonados renacentistas.

\subsection{Trazado de lacería con estrellas}

La estrella de ocho puntas que se usa en carpintería de armar se construye con cintas entrelazadas a partir de elementos lineales de madera escuadrada que conformarán una estructura resistente. Las cintas se obtienen en todo caso mediante una rotación (figura 2). Este diseño sencillo, se elabora dentro de dos metodologías de trazado que obedecen a principios geométricos totalmente diferentes: los trazados de estrellas dentro de una estructura reticular ortogonal y las ruedas. El trazado de ruedas y su organización dentro de la geometría plana de las armaduras está ampliamente explicada por Enrique Nuere (4, p. 225-242). Brevemente, se trata 


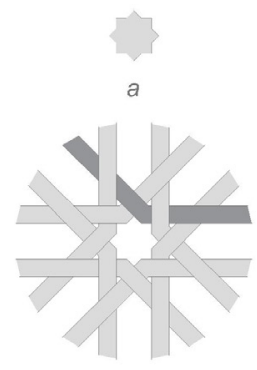

Ángulos a $90^{\circ}$

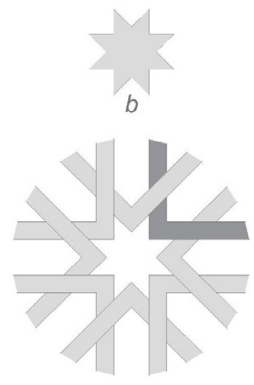

Ángulos a $45^{\circ}$

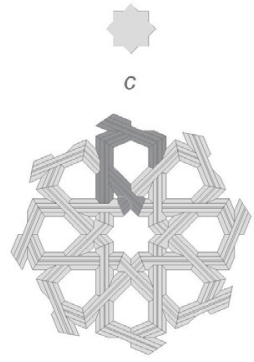

Rueda de 8.
Figura 2. Tipologías de estrellas y rueda de ocho.

de ruedas cuyas cintas radiales se conectan con otras ruedas secundarias denominadas desculatadas, organizándose en el espacio para crear una trama que puede contener dos tipologías de ruedas. La organización de las ruedas se ajusta a un trazado reticular tanto en composiciones ortogonales como no ortogonales.

Resulta interesante como hipótesis analizar la carpintería de armar toledana, de difícil datación, como el posible producto de la industria de aquellos que habitaron la ciudad en épocas tempranas. Conviene descartar la ocupación romana por sus estructuras de cerchas, pero podría valorarse al menos como antecedente en lo que a traza y construcción se refiere, la ocupación visigoda entre el 533 y el 711, y la ocupación del Califato de Córdoba, y la posterior Taifa de Toledo desde 1037 y hasta el 1085. Sin embargo, esta consideración no es el objeto del estudio en tanto no se cuente con métodos de datación absoluta.

En cuanto a la abundancia de la estrella de ocho puntas en las armaduras toledanas a lo largo de la historia y a la superioridad numérica de la composición en retícula ortogonal frente a la composición con ruedas desculatadas resulta elocuente. Sólo dos trazados se generan con ruedas diferentes de las ruedas de ocho. Por otra parte sólo 3 armaduras se componen con tramas de ruedas y sus desculatadas y ambas son ataujeradas.

Pese a la aparente sencillez inicial, la estrella de 8 sobre estructura reticular ortogonal ha generado una variedad de soluciones asombrosa al dotar de complejidad a las armaduras apoyándose en el conocimiento estructural, constructivo y decorativo.

\subsection{Objetivos y método}

Se aplica el método de estudio de casos. Analizadas las principales armaduras de lazo de Toledo se realiza un análisis global que permite la definición de trazas típicas Toledanas, así como profundizar en los sistemas estructurales y en las relaciones entre las diferentes tipologías de traza y su construcción.

\section{TIPOLOGÍAS DE TRAZADO CON ESTRELLAS DE OCHO EN LA CIUDAD DE TOLEDO}

El interés por la estrella de ocho en la carpintería de armar toledana abarca un amplio espectro que incluye la rueda pintada sobre la madera, pasa por la rueda inserta en la estructura con capacidad portante, y se usa como elemento decorativo de madera superpuesto a la estructura portante. Estas opciones se repiten y combinan desde la Edad Media hasta el Renacimiento.

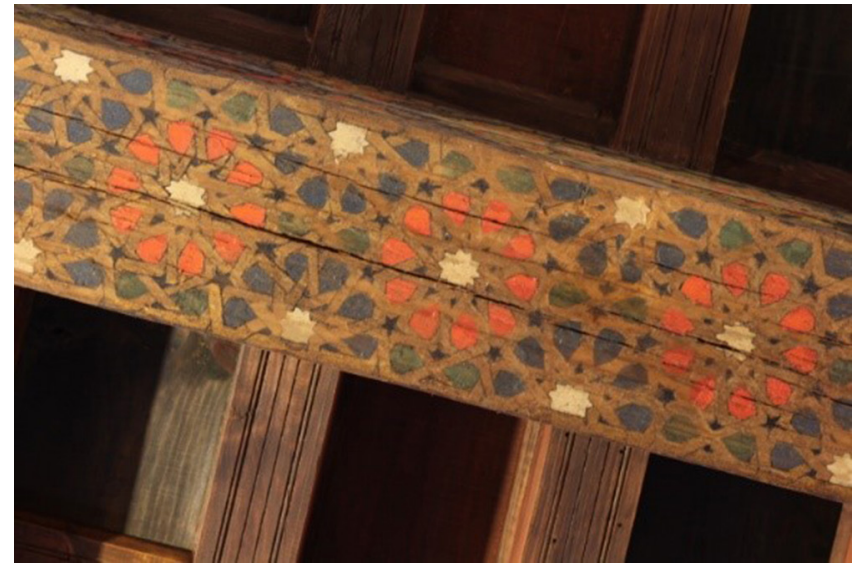

Figura 3. Lacería policromada sobre la madera en el Palacio de Fuensalida, Toledo.

La traza de estrellas, cuando éstas son pintadas, se realiza con plantillas de dibujo que se rellenan con color (figura 3). Por el contrario, el trazado en madera se realiza mediante el uso de cartabones, lo que resulta una constante en la carpintería de armar toledana y nacional y cuyo uso describirá López de Arenas en su Tratado de la Carpintería de lo Blanco, recuperado y explicado por Enrique Nuere (4, p. 231-237).

Sin embargo, las armaduras alcanzan un mayor interés en la traza con la estrella de ocho, cuando ésta se encuentra formando parte del entramado estructural. En este caso la rueda se forma con los elementos portantes, pares o nudillos, y por los peinazos. De su combinación nace una retícula que se aprovecha para insertar diseños que se fundamentarán en los lazos continuos que se entrecruzan y en las estrellas de ocho puntas que aprovechan el hueco dejado por la trama estructural.

La lacería cumple la regla de "corta y pasa”, es decir una cinta pasa por encima de aquella con la que se cruza, y en el siguiente encuentro pasa por debajo. Es posible lograr que las cintas cumplan esta regla gracias a que las trazas se generan por rotación. La generación de estas trazas se logra a partir del giro de un cuadrado sobre sí mismo 45 grados.

\subsection{Trazado con una estrella}

En la Iglesia de Santiago del Arrabal (figura 4) encontramos la traza de una estrella que se ajusta al ancho de la calle, don-

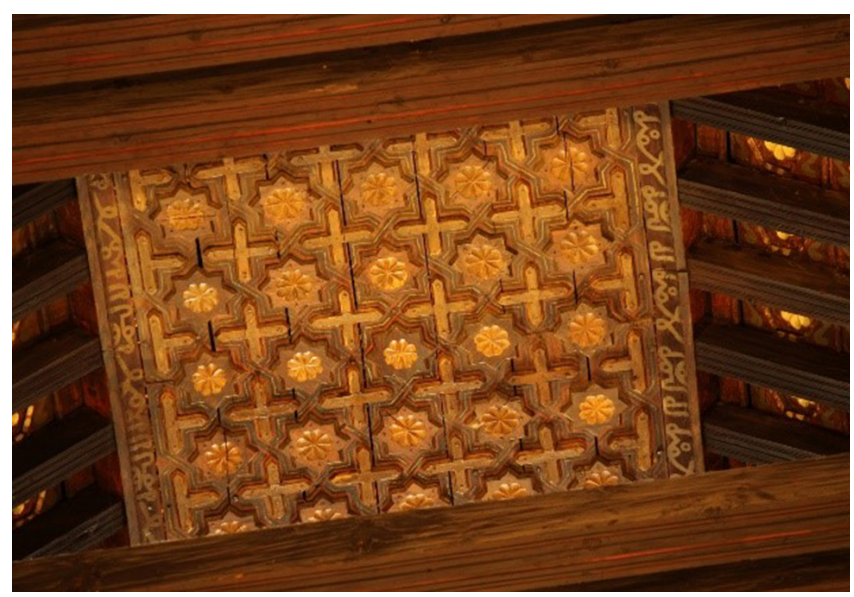

Figura 4. Almizate de la Iglesia de Santiago del Arrabal, Toledo. 
de cada espacio es ocupado por una estrella de ocho puntas, manteniendo la trama de nudillos y peinazos en el diseño a través de crucetas. Este almizate es el único que aprovecha la trama estructural para formar su diseño sin hacer uso de los huecos que quedan entre la madera limitándose a un ataujerado superpuesto. El dorado, muy probablemente con pan de oro, sigue sorprendiendo aún hoy por la luz que refleja.

\subsection{Trazado con dos estrellas}

La traza de dos estrellas se forma por la combinación de la primera estrella de ocho (fig 2a), con la segunda estrella extendida (fig 2b). La retícula que forman ambas estrellas a "calle y cuerda", se completa con peinazos. Los lazos cambian de estrella con un cruce tanto en la vertical como en la horizontal.

Una particularidad de esta traza es que, si bien la estrella se genera por la rotación del cuadrado de 45 grados sobre sí mismo, al incorporar el lazo y los cruces, en una dirección se ajusta perfectamente al esquema de "calle y cuerda", mientras que, si hacemos una rotación de 45 grados, el lazo genera la necesidad de doblar la estructura, lo que podemos comprobar en los paños a 45 grados de los testeros de la Sinagoga del Tránsito (figura 5).

\subsection{Trazado con estrellas de ocho y lazo de cuatro}

Esta traza se forma sobre una estructura a "calle y cuerda", con una retícula de estrellas de ocho puntas separadas entre sí la distancia de un peinazo. Entre cada dos estrellas, los lazos se entrecruzan formando un nudo de transición o lazo de cuatro.

Esta trama se emplea en los almizates de la Iglesia de Santa Clara (figura 6), tanto en la nave principal como en la nave de la epístola. También se emplea en los paños de Santas Justa y Rufina o del Hospital de Santa Cruz, donde se emplean nudos de enlace en la proximidad del almizate, en la base y en el centro del paño.

Un caso particular de la traza con estrella de ocho y lazo de cuatro, se encuentra en la Iglesia de las Santas Justa y Rufina donde se plantea insertar una rueda en el eje del almizate.

Para que se pueda introducir los azafates harpados completos, se recurre a doblar el nudillo y por tanto los pares (figura 7). Se trata de un intento muy interesante y complejo, que en mi opinión quedó interrumpido en favor de una traza más sencilla, especialmente en la ochava, no llegando a completarse ninguna de las dos propuestas.

\subsection{Trazado con ruedas y sus desculatadas}

Curiosamente, en la ciudad de Toledo, si buscamos ruedas de lazo con definición radial, es decir, con ruedas que desculatan en otras ruedas, sólo encontramos tres ejemplos.

Dos de ellos se colocan sobre alfarjes de reducidas dimensiones. Uno de ellos, el alfarje del saloncillo de la Casa de Mesa (figura 8), es un ataujerado superpuesto de ruedas de 9 y 12, sobre trama triangular muy ajustada a la planta de la sala. Las ruedas de 9 desculatan en ruedas de 12 que se sitúan en la calle central y el límite de la sala, aprovechando la rueda par para generar una calle de cierre en la traza. El artesano resalta sinos y azafates con un alto-relieve dejando en segundo plano el lazo.

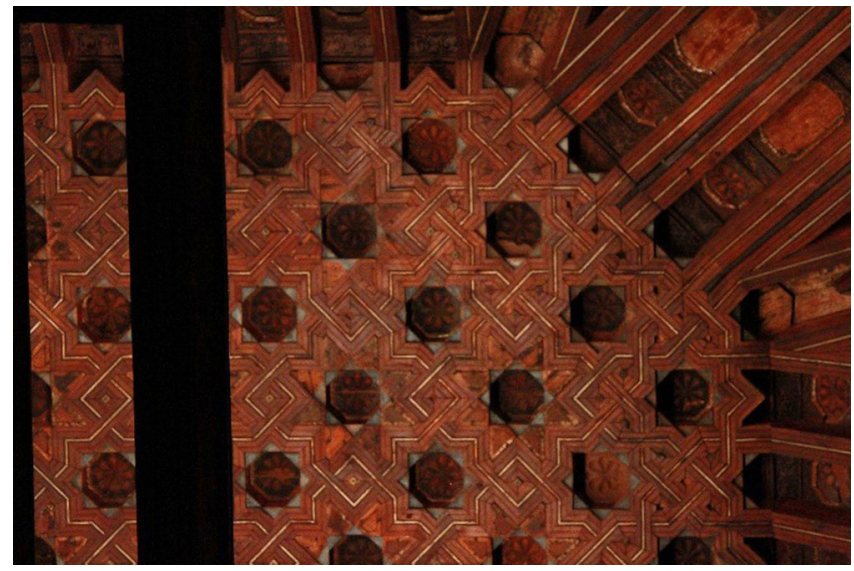

Figura 5. Almizate de la Sinagoga del Tránsito, Toledo.

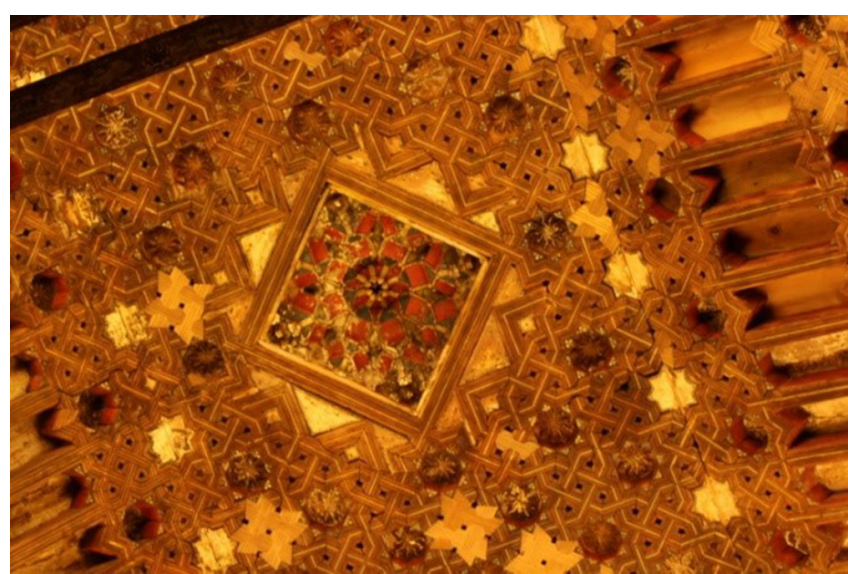

Figura 6. Almizate de la Nave de la Epístola de la Iglesia de Santa Clara, Toledo.

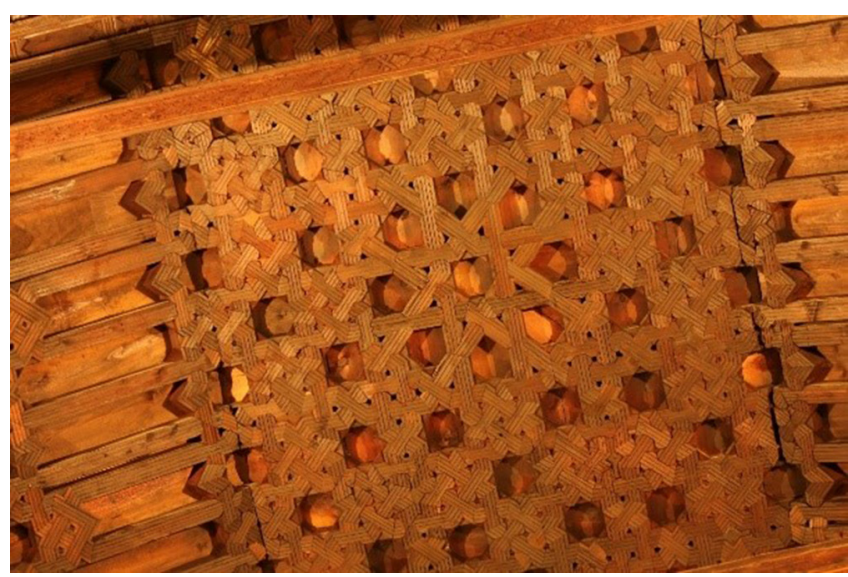

Figura 7. Almizate de la Iglesia de las Santas Justa y Rufina, Toledo.

Otro ataujerado plano de pequeña dimensión es el del Convento de Santa Úrsula. Se trata de ruedas de ocho que desculatan en ruedas de 16 con mocárabes de diferentes tamaños incorporados en los sinos externos de ocho y en el punto central de la sala. En este caso, la traza se desdobla en la pared, lo que, por poco habitual, hace pensar en una posible reutilización del elemento decorativo y en todo caso, en el tratamiento de la madera como decoración superpuesta a un elemento estructural. El tercer caso es la traída por el Marqués de la Vega Inclán para la Casa del Greco, donde se resuelve una ochava con ruedas de 10. De nuevo se trata de un ataujerado. 


\subsection{Trazas con cuartillejos}

El cuartillejo, definido textual y gráficamente por Fray Andrés de San Miguel y dibujado por López de Arenas, se describe como un módulo cuadrado que se compone de cuatro cuartos de rueda de ocho, haciendo coincidir las esquinas del módulo con los centros de los sinos. Cuando los azafates se adaptan a la trama reticular, se modifican y se denominan azafates harpados (figura 9). Es el caso de la escalera del Hospital de Santa Cruz y de parte de la Iglesia de las Santas Justa y Rufina.

El cuartillejo se emplea abundantemente en Toledo, donde se adapta a otras ruedas diferentes de la de ocho y a otras posiciones dentro de la estructura como se analizará más adelante, lo que, unido a sus variaciones ya mencionadas, hace de él un concepto que se define en cada ocasión.

Hay que destacar que no se emplean cuartillejos en las armaduras tempranas como las de la Iglesia de Santa Clara o la sacristía del Convento de Santa Isabel.

\section{TIPOLOGÍA ESTRUCTURAL Y CONSTRUCTIVA}

Las obras de construcción se enfrentan a problemas atemporales que definen los procesos que los solventan. Considerando el trazado una actividad previa a la construcción, la hiciese o no el mismo carpintero, el proceso de construcción conlleva encontrar la madera que permita realizar el encargo, el escuadrado y azuelado de la misma, el trabajo de carpintería sobre la madera que genere las uniones y la decoración, la aplicación de acabados (aceitados, protecciones, pinturas, dorados...), su montaje en obra, el cierre superior de la cubierta (sobrecubierta, sotopares, aleros, morteros, teja...) y la decoración interior de la misma.

Antes de analizar los tipos constructivos de almizates y faldones se definirán ciertos términos:

\subsection{Armaduras de par y nudillo}

Son aquellas armaduras de cubierta en las que los empujes horizontales de los pares sobre los muros son contenidos por los estribos que se mantienen estables sobre el muro por la acción de los tirantes. La función de los nudillos es la de acortar la luz del par y estabilizar. Pueden tener o no hilera

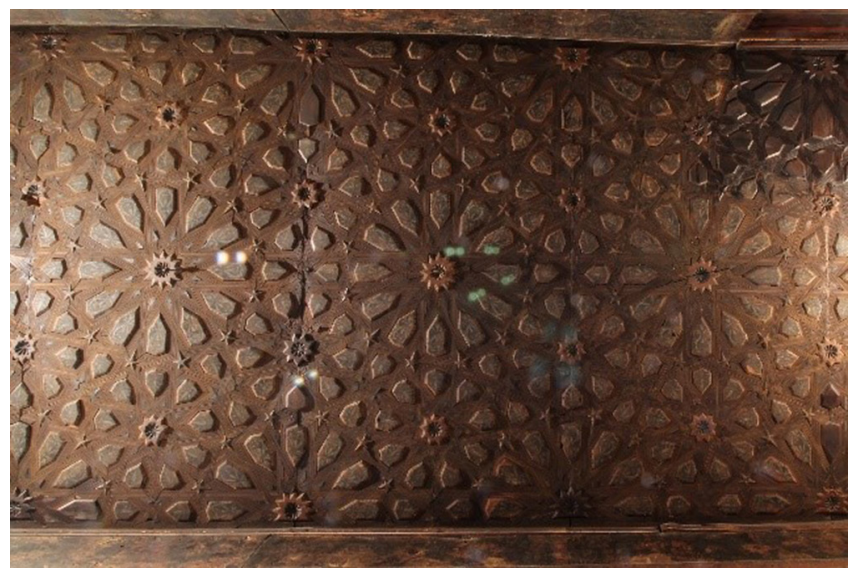

Figura 8. Ruedas de lazo de ocho en el alfarje de la Casa de Mesa, Toledo. y los pares pueden llegar hasta la posición de la hilera o interrumpirse al superar el nudillo. Este es el caso de los pares de los testeros, aunque se encuentran armaduras con pares cortados, lo que en muchas ocasiones obedece a la colocación de una sobrecubierta posterior que necesita el hueco para sus tirantes.

\subsection{Armaduras apeinazadas y ataujeradas}

La construcción de armaduras con lazo se realiza de dos formas. Hay armaduras en las que las cintas son elementos estructurales. Estas armaduras se denominan apeinazadas ya que las cintas las forman los pares combinados con otras piezas habitualmente perpendiculares a los pares denominadas peinazos. Las armaduras ataujeradas, por el contrario, se forman por tablas unidas entre sí y reforzadas posteriormente formando un conjunto plano estable con capacidad exclusivamente autoportante. Prieto Vives define el término ataujerado como "techo cuyo adorno se forma con listones clavados, por oposición al apeinazado, en el que el adorno se forma con su misma estructura” (5).

\subsection{Armaduras de cubierta y armaduras autoportantes con sobrecubierta}

Las cubiertas soportan la carga del tablero, del mortero y de la teja, así como las cargas de viento y nieve. Cuando las armaduras de lazo soportan esta carga son necesariamente apeinazadas. Sin embargo, no todas las armaduras apeinazadas tienen función estructural. En ocasiones, se elige colocar una sobrecubierta para mejorar la durabilidad de las armaduras de lazo ya que el camaranchón da la opción de generar una ventilación cruzada que protege la madera. Además, se solía dar en estos casos una protección de yeso posterior a la armadura decorada. Esta protección higroscópica libera a la armadura de los cambios de humedad ambiental e incluso de pequeñas humedades procedentes del desplazamiento de las tejas y así mantiene la madera libre de pudrición hasta el siguiente retejo.

\subsection{La Prefabricación}

La prefabricación es la gran herramienta de la construcción de armaduras de lazo. Los paños pueden construirse y decorarse en el suelo, izándolos en grupos de 4 o 5 calles, tal y como reconocemos en las obras existentes. Para su montaje,

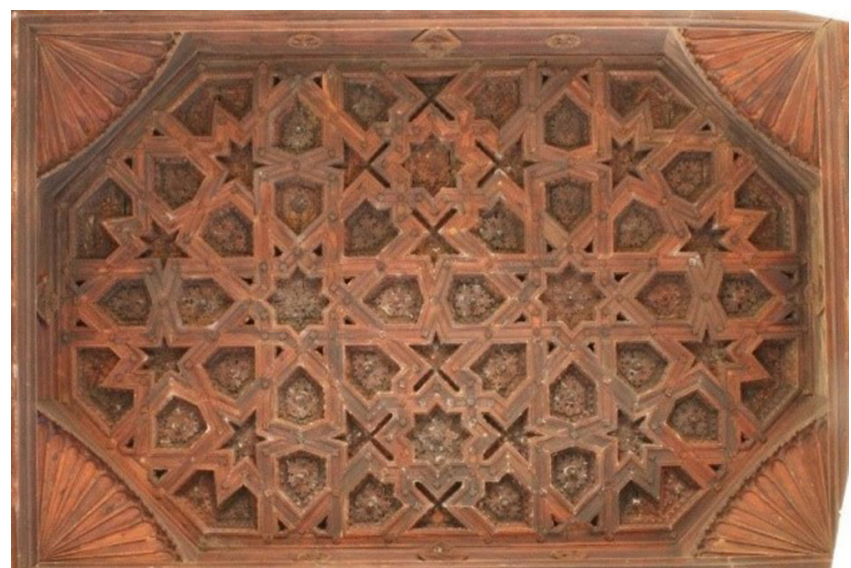

Figura 9. Estructura sobre la escalera del Claustro del Hospital de Santa Cruz, Toledo. 
se enfrentan dos paños y se dejan caer sobre el almizate previamente colocado en su posición con un andamio apoyado sobre los tirantes. ¿Qué hizo posible que se llegase a este eficaz sistema? Sin duda fue la combinación de dos cuestiones. La primera era el control de las dimensiones de los faldones gracias al sistema de cartabones. Sin un control exquisito de estas dimensiones, los errores habrían imposibilitado el uso. De entre todas las posibles uniones, algunas de las que se recogen en la tesis doctoral sobre la región leonesa (6), se opta en la prefabricación por la unión a garganta y quijera. El manejo de elementos de grandes dimensiones es posible, porque la unión permite un montaje sencillo frente a otras uniones que habrían imposibilitado el proceso.

La unión garganta y quijera se ha encontrado realizada a $1 / 3$ de la sección en armaduras tempranas como la de la Iglesia de Santa Clara de Salamanca, sin embargo, López de Arenas escribe "Ternan de grueso las quixeras una quinta parte de su grueso, y esa quinta parte se quitará al alfarda en la garganta por los lados y lo mismo por el pecho."(4). Parece que esta unión ha estado en la carpintería española acompañando a la lacería y a las armaduras de par y nudillo, y ha evolucionado a mejor pasando de debilitar el par cajeando 2/3 de su sección a cajear 2/5 de la unión como dice López de Arenas y como encontramos en numerosos ejemplos de la carpintería española.

La carpintería de armar toledana en cubiertas gira en torno al sistema estructural de par y nudillo que se va desarrollando en función de las necesidades de cada obra. La interacción de procesos de construcción, sistemas estructurales y trazas genera un corpus de sistemas constructivos muy rico en la ciudad de Toledo, que bien parece un campo de pruebas de la carpintería de armar en su acercamiento a una lacería más compleja que determinará los sistemas óptimos y sus reglas.

Resulta imposible a día de hoy saber la fecha exacta en la que se realizaron las armaduras, lo que resultaría interesantísimo cara a entender la evolución temporal de la carpintería de armar Toledana y su relación con el resto de la carpintería nacional, sin embargo sí se puede analizar la evolución de los sistemas constructivos hacia su complejidad y su eficacia, mientras quedamos a la espera de que la dendrocronología o el carbono 14, las obras de restauración y la arqueología aporten datos que encajen temporalmente dichos sistemas.

\section{CONSTRUCCIÓN DE ALMIZATES}

El almizate es el "paño horizontal plano formado por el conjunto de los nudillos en las armaduras de par y nudillo" (4, p. 264).

\subsection{Almizate entablado}

Es aquel en el que un tablero clavado sobre la cara posterior de los nudillos no permite ver la hilera. Esta tablazón se justifica de varias formas: la tablazón del nudillo oculta descuadres de la hilera o de la unión de parejas de pares; la tablazón oculta la no continuidad de los pares; o bien la tablazón estabiliza los nudillos uniéndolos entre sí a la distancia necesaria para que el encaje de los pares sea exacto.

$\mathrm{Al}$ hacer cubiertas a cuatro aguas, la carga de los pares del testero sobre el último nudillo produce una flexión sobre éste

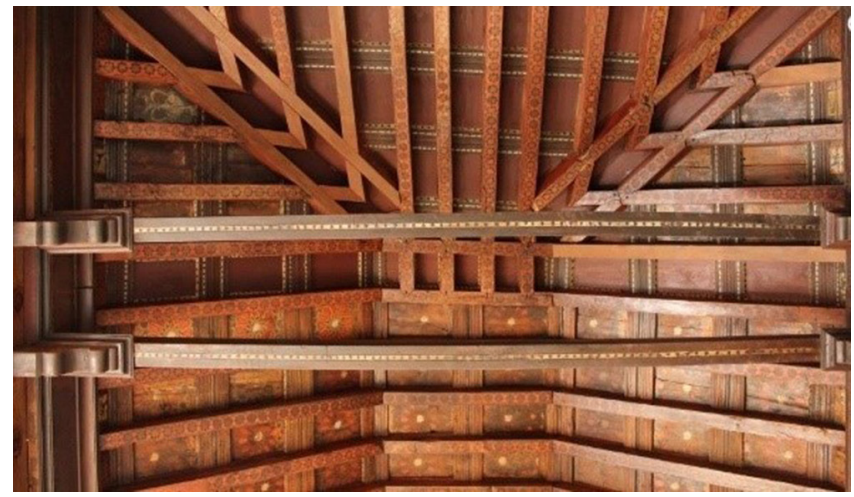

Figura 10. Biblioteca del Palacio de $\mathrm{D}^{\mathrm{a}}$ Inés de Ayala.

que se minimiza repartiendo la carga sobre varios nudillos y formando una pequeña retícula entre los primeros 2 o 3 nudillos (figura 10). Este avance de retícula evoluciona en una retícula por tramos o continúa en todo el almizate, que, ya sin trabajo estructural, asume la nueva función de incorporar decoración.

\subsection{Almizate ataujerado}

Quizá el sistema más sencillo de decorar el almizate sea el empleado en la Iglesia de Santiago del Arrabal (Figura 4), donde, el posicionamiento de nudillos en el proceso constructivo de la armadura se logra con un plano continuo ataujerado. El tablero ha sido prefabricado, como nos indican la clavazón de éste a los nudillos, y se aprovecha para recoger la decoración. Ésta no es otra que la trama de estrellas de ocho puntas que se ajusta perfectamente a la sucesión de nudillos. Las cintas cumplen la regla de "pasa y corta", y se aprovechan los sinos para alojar decoración (flores de ocho puntas y cruces). Por la posición de los clavos se puede ver que las tablas van clavadas sobre los nudillos, uno sí y uno no, y se clava también el perímetro del tablero. La decoración va clavada con clavos de menor dimensión. Si bien los clavos menores están posicionados en el lugar de hipotéticos peinazos, muy probablemente éstos no existan y sí existan tablas menores en la dirección perpendicular al tablero que estabilicen el conjunto formando un conjunto rígido más fácil de manejar al elevarlo. La policromía pasa por encima de los clavos. No se da una gran importancia al ancho de la tabla, pero sí a su longitud formando un tablero regular, que no termina sobre el nudillo sino entre éstos, quizá para admitir errores de replanteo y ajustar más los tableros entre sí. De hecho, no se aprecia separación entre las testas de las tablas. Los octógonos se encuentran clavados con 4 puntas en sus esquinas, salvo donde se unen los tramos de almizate. Muy probablemente hayan sido clavadas desde la cara posterior durante el montaje ya que se intuyen pequeños incisos de puntas sin llegar a deteriorar el dorado.

Las tablas han mermado dejando espacios entre ellas y marcando su organización. Muy posiblemente se hayan pintado y dorado directamente sobre una imprimación de cola sin utilizar un aparejo de yeso previo que pudiera haber evitado que se marcasen tanto las uniones entre tablas, tal y como se resolvió en ocasiones en la techumbre de la Catedral de Teruel. "Aparejo es la denominación que aparece de forma generalizada en los documentos españoles. Se refiere a la preparación blanca, generalmente de yeso, para la pintura y la aplicación de láminas de metal”(7). 


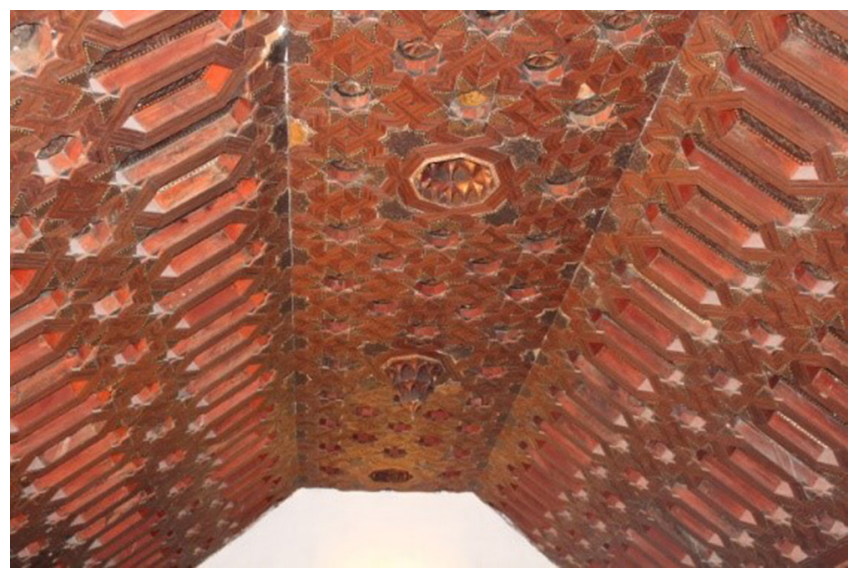

Figura 11. Sacristía del Convento de Santa Isabel. Toledo.

Sin tener clara la fecha de construcción de Santiago del Arrabal, su primera mención es en 1114 y, con independencia de su fecha cierta de fundación, de entre las Iglesias existentes tras la reconquista en 1085 , "tal vez sea la que mejor ha conservado su estructura originaria” (8).

El sistema habría posibilitado la talla de nudillos y peinazos lo que respondería a la lacería convencional, pero se emplea un ataujerado sin resolver correctamente los problemas de la base pictórica. Conceptualmente y apoyados en su posible fecha de construcción, parece una armadura temprana que aprovecha de forma natural los elementos lo que la hace de ella una perfecta candidata para realizar estudios dendrocronológicos.

\subsection{Almizate apeinazado con dos estrellas}

En la sacristía del Convento de Santa Isabel (figura 11) o en la Sinagoga del Tránsito (figura 5) se emplea este sistema de peinazos y taujeles con la traza de dos estrellas. En ambas, el almizate se compone de nudillos y peinazos que forman el conjunto estructural rígido. Se aprovecha la opción tridimensional que ofrece la estructura alojando conjuntos decorativos propios o incorporando mocárabes o pinjantes.

Al no tener acceso a las armaduras por su cara posterior, se hace una interpretación de las mismas. Los nudillos se conectan en grupos mediante peinazos perpendiculares que habitualmente se sujetan con uniones a caja y espiga. La trama de peinazos es tan densa que muy probablemente se alternen peinazos estructurales con tablas clavadas en L de forma que se permita la clavazón de los taujeles sin sobrecargar la armadura. A partir de la clavazón en los laterales de las cajas rehundidas de las estrellas se propone que se haya formado con artesones armados en las esquinas con los triángulos necesarios para formar la estrella en volumen, y cerrados por su parte superior con una tapa que contenga el bajo relieve de la flor.

En la Sacristía de Santa Isabel de los Reyes (Figura 12) la trama de dos estrellas incorpora mocárabes. El ataujerado se compone pintando un falso saetino punteado en las estrellas a 45 grados mientras que las cintas se forman clavando tablillas sobre nudillos y peinazos. Se aprecia en las uniones la existencia de una capa de yeso posterior de protección. Se trata de un trabajo finísimo (figura 11), donde apenas se aprecia la clavazón escondida en el agramilado. Hay un gramil grueso central negro, y dos laterales muy finos a cada lado, uno negro y uno rojo. La unión de los gramiles es extraor-
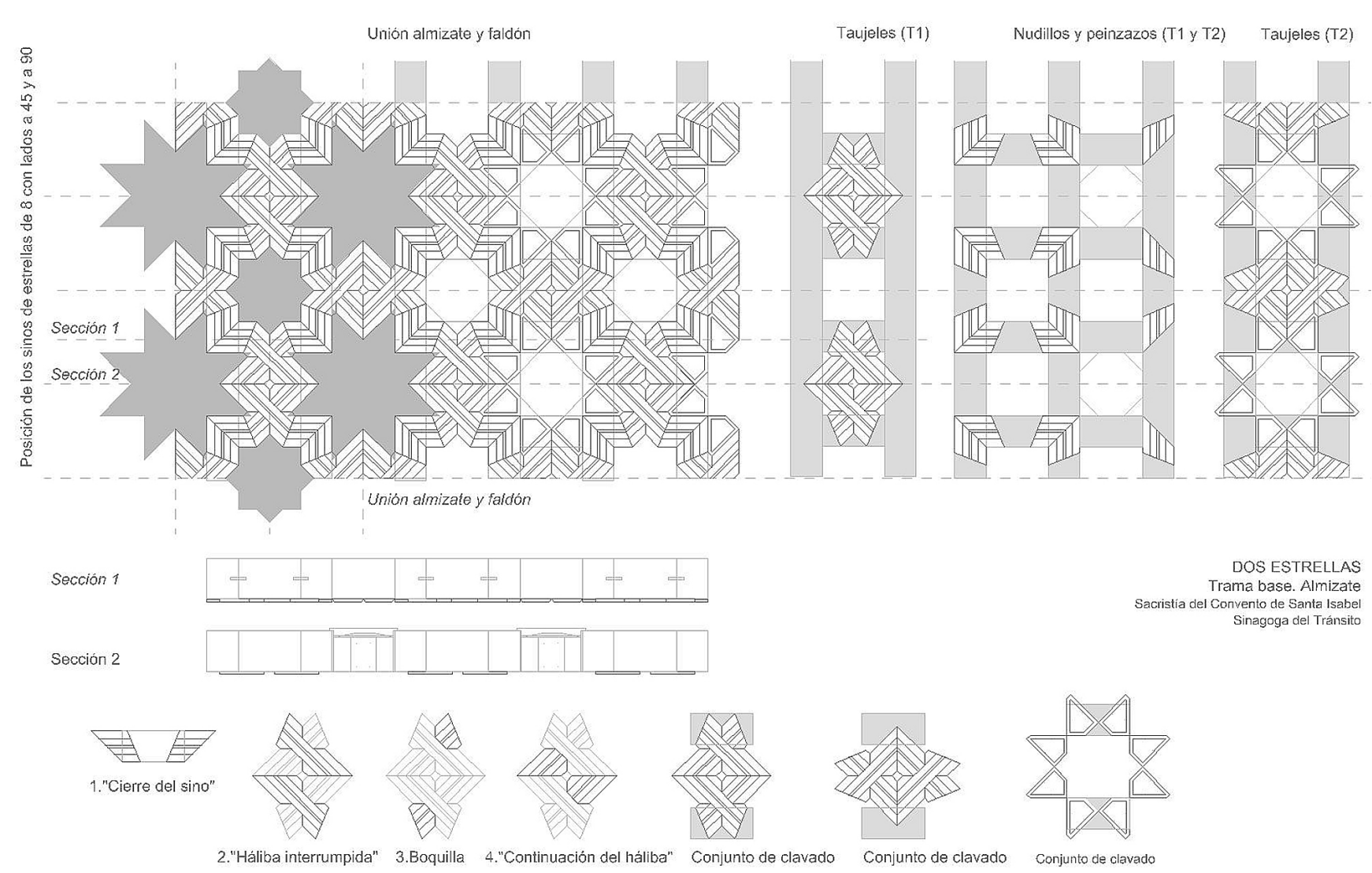

DOS ESTRELLAS Trama base. Almizate Sinagogal drabusito

Figura 12. Construcción de un almizate apeinazado con traza de dos estrellas. Sacristía del Convento de Santa Isabel. 
dinaria considerando que sólo tras un perfecto cepillado se puede ejecutar un agramilado tan fino.

\subsection{Almizate apeinazado con estrellas de ocho y lazo de cuatro}

En las armaduras de la Iglesia del Convento de Santa Clara (figura 13), en las ochavas del Taller del Moro o en la cubierta del Palacio de Fuensalida, encontramos la traza de estrellas con lazo de cuatro que es la más habitual en Toledo.

Su complejidad visual contrasta con el escaso número de formas que emplea. También se organiza sobre una retícula cuajada de peinazos o falsos peinazos sobre los que se clavan los taujeles. Muy interesante es la clavazón que alterna clavos de mayor entidad para los taujeles mayores siempre sobre su diagonal y ocultos, con clavillos menores en las pequeñas piezas.

\subsection{Almizate apeinazado con doble lazo de cuatro}

La Iglesia de las Santas Justa y Rufina, documentada en 1536 tras un incendio y un cambio de orientación de la Iglesia en obras de Alonso de Covarrubias en 1530 (3, vol. I, p. 263-275), resulta particular desde el punto de vista de la posible prefabricación. En ella, se prueban dos posibles trazados no completándose la nave con ninguno. Se emplea una solución apeinazada con cuartillejos (figura 14) y una particular solución que denomino de "doble lazo de cuatro".

Este último es el más complejo de los dos trazados de la Iglesia de las Santas Justa y Rufina y es una variante de la traza de estrellas de ocho y lazo de cuatro que no se llegó a completar (figura 7). La incorporación de una rueda al eje del almizate ya explicada en el apartado 2.3., genera una solución constructiva muy interesante. Se traslada el concepto de las limas moamares al almizate, doblando nudillos parcialmente y por tanto pares en favor de la prefabricación.
La sencillez constructiva es máxima ya que los taujeles y peinazos entre ambos nudillos también podrían estar prefabricados. Fue un intento inconcluso en el que el carpintero particularizó abriendo una vía de desarrollo de traza y construcción, aunque al llegar a la ochava la solución habría sido muy compleja.

\subsection{Almizate apeinazado con cuartillejos}

El más sencillo de los dos trazados existentes en la Iglesia de las Santas justa y Rufina y muy empleado en Toledo, es el organizado por cuartillejos, una estructura prefabricada en la que un pequeño grupo de piezas se repite facilitando así el proceso constructivo. Esta sencillez en el trabajo carpintero, se complica frecuentemente con otros recursos como pueden ser pechinas como en la escalera del Hospital de Santa Cruz, o elementos decorativos específicos. Los cuartillejos, ya analizados por Fray Andrés de San Miguel (9) y desarrollada su secuencia constructiva por Javier de Mingo (10), habitualmente se realizan con estrellas de ocho. Su sistema constructivo desarrolla un marco estructural. Sobre este marco se desarrolla la traza de sinos, aspillas y azafates harpados centrales. Son rígidos y prefabricados, lo que permite un sencillo montaje y manipulación. Su particularidad como sistema se basa en que el cuartillejo, habiendo adquirido rigidez como conjunto, permite quitar nudillos. Este concepto de paño rígido, se empleará posteriormente en el desarrollo de la lacería con ruedas desculatadas cuya traza llegará a no poder ajustarse más que a las limas debiendo conformar paños estables.

\subsection{Ruedas centrales}

En Toledo, los cuartillejos no se limitan a las estrellas de ocho puntas. Es frecuente ver almizates coronados con una rueda única que se enmarca relacionándose con los faldones y que denomino rueda central. La rueda se rodea por líneas con sinos y azafates, habitualmente ubicado el eje de los sinos en el pliegue entre faldón y almizate. Esta estructura
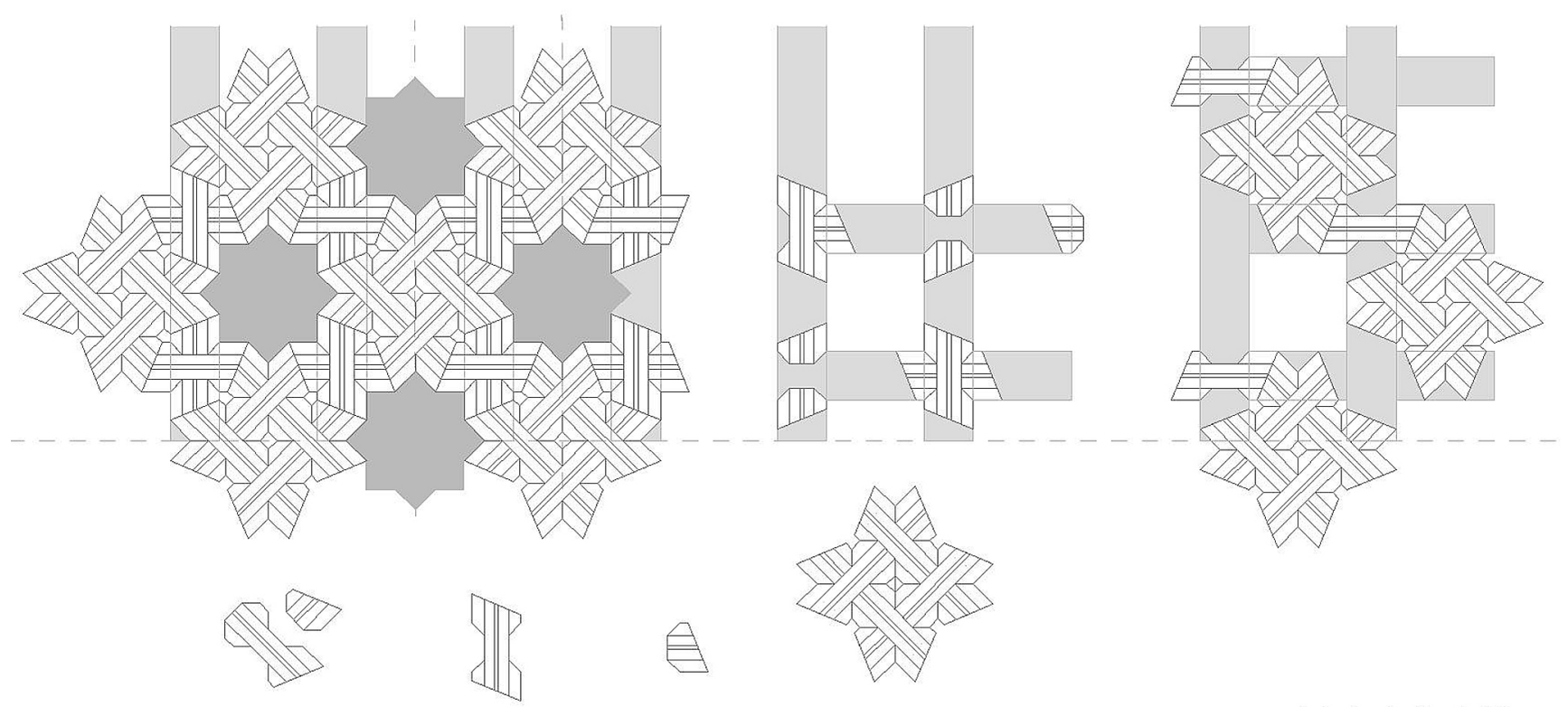

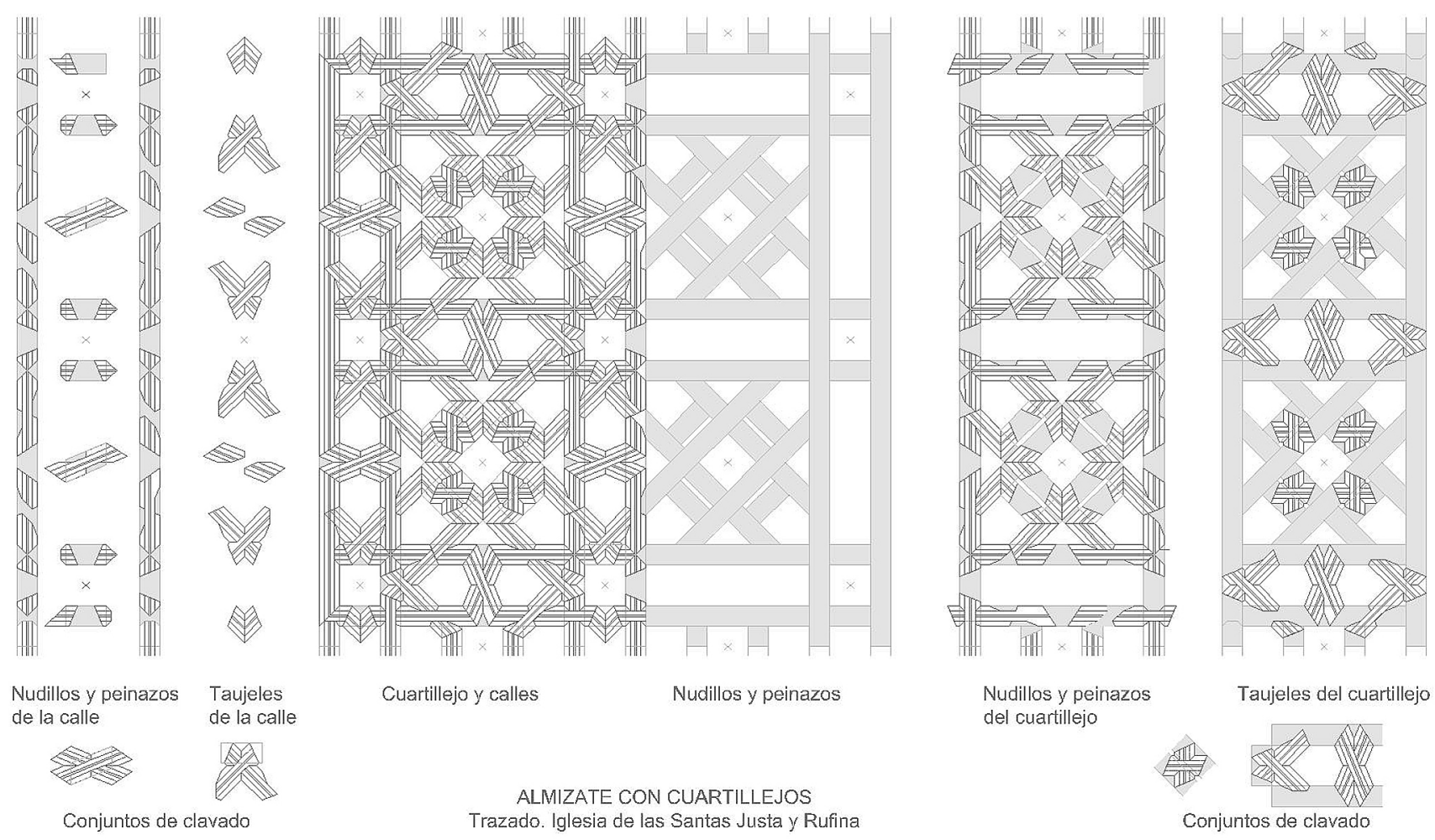

Figura 14. Construcción de un almizate apeinazado con cuartillejos. Iglesia de las Santas Justa y Rufina.

posibilita una relación natural con los faldones. Este es el caso de la Iglesia del Convento de Santa Isabel, del rincón del claustro de San Juan de los Reyes o del forjado del Palacio de Benacazón.

\section{CONSTRUCCIÓN DE FALDONES}

Los faldones comparten trazas y sistemas constructivos con los almizates, sin embargo, tienen ciertas particularidades de las que, en Toledo, surgen nuevos sistemas constructivos en toda su complejidad.

\subsection{Cubiertas a dos aguas. Limas bordón y moamar}

Un número elevado de las armaduras toledanas, en particular aquellas que cubren edificios con indicios de haber sido anteriormente mezquitas, tienen armaduras a dos aguas, con faldones decorados con tableros a cinta y saetino y policromía. Los faldones evolucionan constructivamente cuando las cubiertas requieren verter sus aguas a más de dos vertientes y aparecen las limas bordón y moamar.

López de Arenas explica en el capítulo ocho de su tratado como establecer el largo de las péndolas en las limas bordón pares y nones. En Toledo encontramos limas bordón en pequeñas armaduras como la cubierta de la sala sur del Palacio de $\mathrm{D}^{\mathrm{a}}$ Inés de Ayala. Que las péndolas sean pares o nones depende del mejor ajuste al ancho de la planta, pero se vuelve una cuestión primordial cuando se decora el almizate ya que las péndolas pares permitirán que haya un eje longitudinal central donde encajar elementos centrales como puedan ser estrellas o mocárabes; mientras que las péndolas nones fuerzan a diseños asimétricos. En Toledo se encuentran almizates con huecos impares. Parece que el gusto por la simetría prima incluso en ausencia de elementos centrales de adorno. De igual modo, se usan las limas moamares como signo de calidad. Tal es el caso de la armadura del Taller del Moro.

\subsection{Faldón prefabricado}

La prefabricación, se particulariza en los faldones para lograr la estabilización del conjunto. Para ello, los peinazos se colocarán, al menos, en la base del faldón y próximos al encuentro con el almizate. El posicionamiento de los sinos en las uniones entre paños no sólo es un recurso de encaje de la traza sobre una superficie que se pliega, sino que contribuye a la perfección a mejorar su rigidez ya que los sinos se delimitan por peinazos que atan la parte superior del paño prefabricado. Con el incremento de la luz de la sala, los paños de faldón van necesitando mejorar su rigidez con sinos intermedios o doblando las franjas de sinos concatenados en diferentes posiciones creando una riqueza de soluciones carpinteras a partir de las trazas básicas (figura 15).

En Toledo, el concepto del cuartillejo como conjunto prefabricado, trasciende su configuración con rueda de ocho, y lo encontramos ubicado, esta vez en los faldones de la Casa de Mesa (figura 16), donde se emplean conjuntos que contienen ruedas de 12, y se emplean indistintamente en el almizate, y en todos los faldones de esta cubierta de 7 paños. Se omiten pares en favor de un conjunto rígido, ligero y prefabricado.

\subsection{Faldón ataujerado}

Por último, encontramos en Toledo el ataujerado como solución cuando se requiere minimizar la estructura. Se trata de la capilla del Corpus Christi en la Iglesia de los Santos Justo y Pastor, donde una sobrecubierta permite reducir la estructura portante, lo que se aprovecha para omitir pares y nudillos de la habitual base a calle y cuerda, y construir una armadura ricamente decorada mediante un ataujerado ligero. 


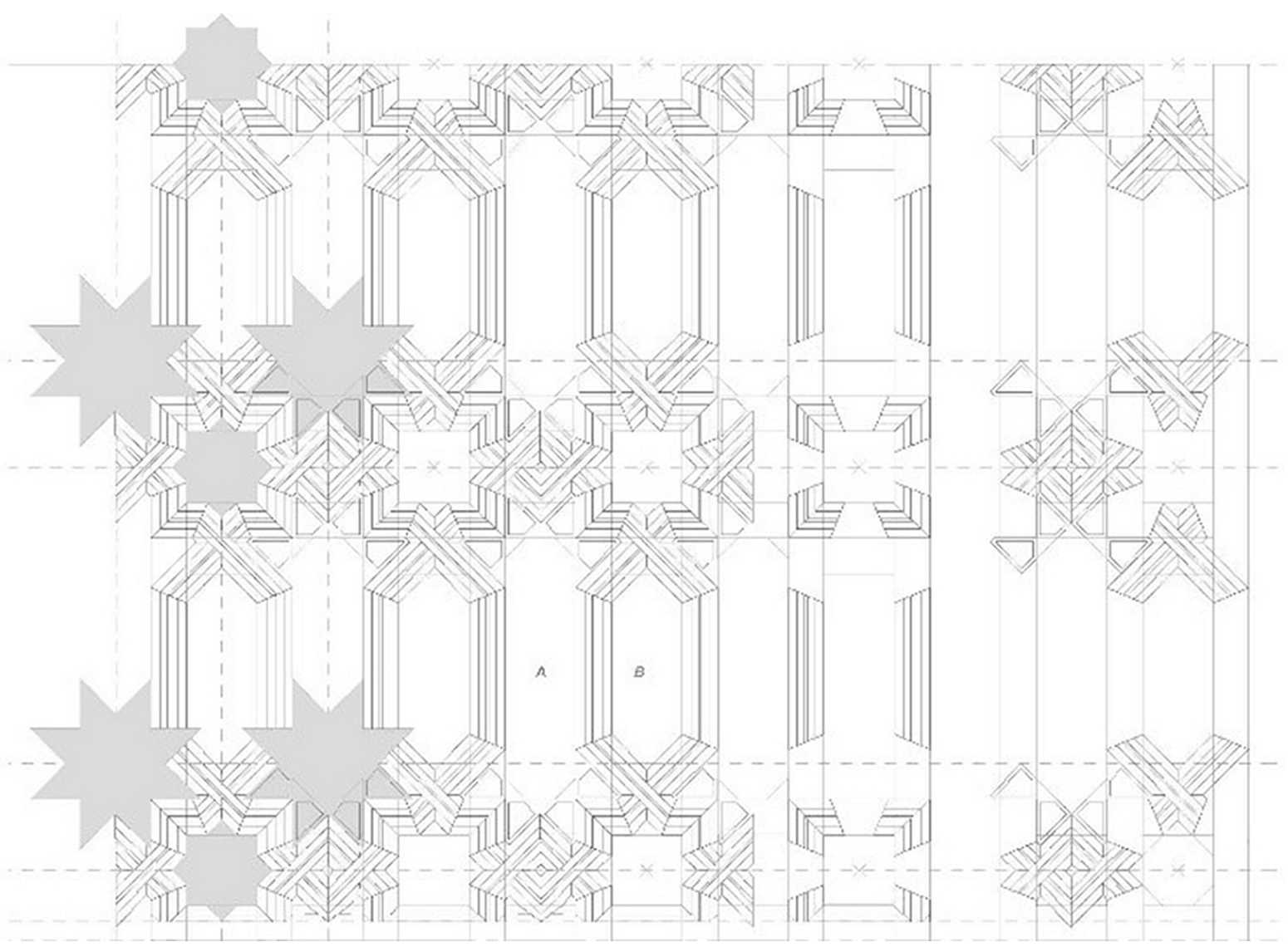

Figura 15. Construcción de faldones prefabricados con lazo en la Sacristía del Convento de Santa Isabel.

\section{ARMADURAS DE SIETE PAÑOS}

Toledo no tiene armaduras de 5 paños, sin embargo, tiene dos impresionantes armaduras de 7 paños, la Casa de Mesa y la Capilla del Corpus Christi. La capacidad técnica para desarrollar estos trabajos hay que buscarla en el bagaje constructivo de la ciudad.

Como aproximación a su estudio podemos apoyarnos en la información de los tratadistas. López de Arenas nos deja una sección de la "Montea de nueve y doze en sinco paños" (11), mientras que en 1699, Rodrigo Álvarez dibuja una sección del "strivamento de la obra fecha en armadura en Arte de cinco paños”.

Las armaduras de 5 paños cuentan en ocasiones con tirantes que interrumpen la continuidad de los faldones. Estos tirantes en ocasiones son de hierro. Sin embargo, otras armaduras de 5 paños prescinden de los tirantes cuando la resultante de cargas entra dentro del muro. Tal es el caso de la Iglesia del Convento de Santa Clara de Tordesillas o de la Capilla de Santiago del Convento de Las Huelgas de Burgos. Ello se consigue por diversos métodos: minimizar la carga de la armadura mediante una sobrecubierta, engrosar los muros o darles continuidad en altura con un trabajo equivalente al de los pináculos sobre los arbotantes, obteniendo una resultante de cargas con mayor pendiente que entre dentro el espesor del muro. La Capilla del Corpus Christi usa todas las estrategias descritas para lograr equilibrar los empujes horizontales de la cubierta (figura 17).

La solución constructiva de los 7 paños se obtiene gracias a la posibilidad de encaje de los paneles sobre los pares con uniones de garganta y quijera en los que las quijeras abrazan por completo al par. Se logra no emplear tirantes sumando los recursos empleados en las armaduras de 5 paños a una

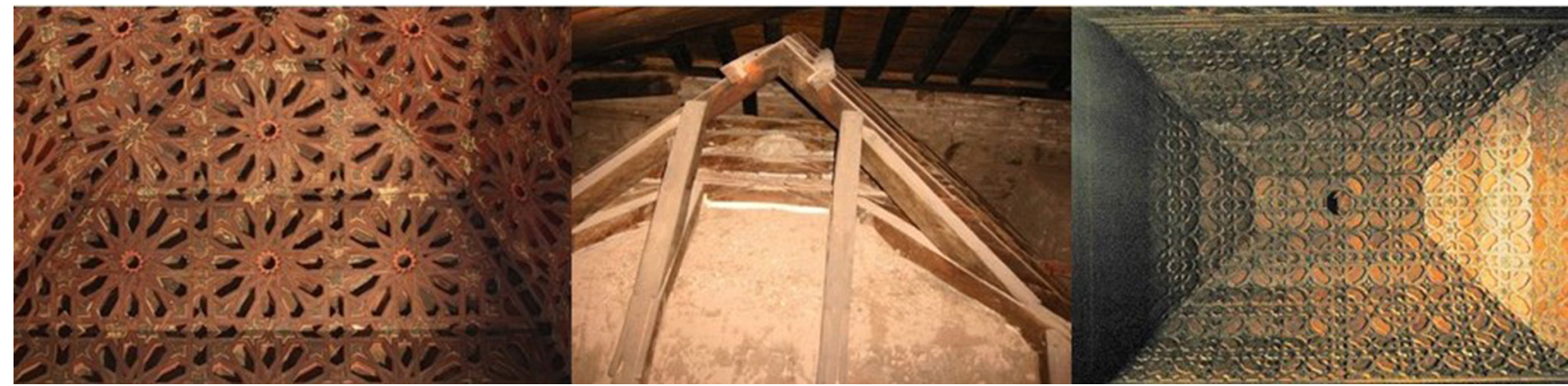

Figura 16. Armaduras de 7 Paños. Casa de Mesa y trasdós e intradós de la Capilla del Corpus Christi en la Iglesia de los Santos Justo y Pastor. Fotos de Enrique Nuere. 
nueva organización estructural que también actúa a favor. Por un lado, la carga de la estructura se minimiza al dejar el panel inferior a una cota más baja, y se reduce el efecto sobre el muro al tomar como par el paño intermedio, y no el que recoge el almizate, así la resultante de cargas tiene mayor pendiente. $\mathrm{Al}$ tener una sobrecubierta se logra obtener un aporte de carga suficiente para compensar los esfuerzos horizontales.

Los pares se unen sin hilera y tienen una guía o regla alta que marca las distancias entre ellos. Analizada la clavazón, la hipótesis de acerca de cómo fue su montaje sería la siguiente: se izaron conjuntos formados por 2 pares con el nudillo y la siguiente estructura de paño por cada lado, ya que en estas piezas parece que la unión garganta-quijera está reforzada por clavazón. Estos conjuntos se alinearían mediante la regleta alta, y ya sobre ellos se clavaría la decoración prefabricada. El último paño no tiene clavazón ya que se podría encajar desde abajo y acuñar sobre un apoyo similar al del par, pero preparado para su alineación. Los tableros se encuentran protegidos por el trasdós por una capa de yeso.

El caso del Salón de Mesa parece ser diferente ya que desde el intradós de la armadura se aprecian peinazos. También se aprecia un mortero blanco que rebosa entre las piezas, que bien podría obedecer a una capa de yeso de protección, lo que indicará la posible existencia de un camaranchón. En ese caso, y, de nuevo dentro del terreno de la hipótesis, la armadura apeinzada sería autoportante por lo que la escuadría de las piezas podría ser reducida. Emplea como configuración geométrica el cuartillejo, en este caso con ruedas de 12, con sus brazos ortogonales acortados. El trazado reticular es muy útil ya que ajusta de forma equidistante en las limas quedando resuelta la calle de limas a la perfección.

\section{CONCLUSIONES}

Toledo muestra una evolución constructiva en la lacería reticular ortogonal que parte de la estrella de ocho, siendo este tipo de trazas casi exclusivas en las armaduras de lazo de la ciudad. Se desarrolla el concepto de prefabricación y dentro de éste, el de cuartillejo. La variabilidad en la traza que encontramos en los cuartillejos, los determina como conjuntos

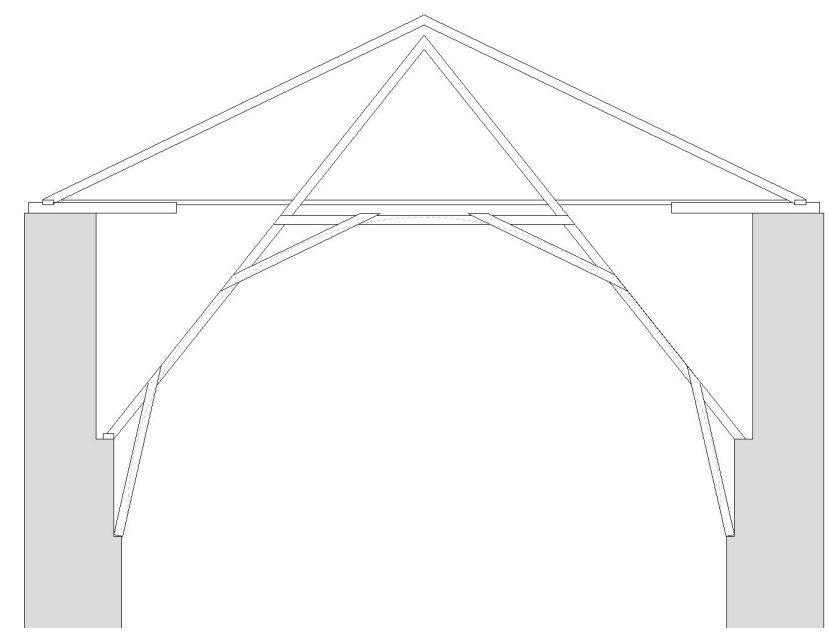

Figura 17. Sección constructiva de la armadura de la Capilla del Corpus Christi.

con la rigidez necesaria para omitir piezas de la estructura base de la armadura. Este concepto permite llegar a las complejas armaduras de 7 paños de la Casa de Mesa y del Corpus Christi.

Toledo también muestra soluciones fallidas intentando aplicar conceptos teóricos válidos que serán retomados en el futuro, como es el caso de intentar duplicar pares de borde de paño prefabricado como si de limas moamares se tratase. Curiosamente en Toledo, la lacería radial de ruedas y sus desculatadas no se desarrolla de forma general, si bien, sí se emplean los ataujerados cuando la traza o la economía estructural así lo aconseja. Es de gran interés la gran evolución de la carpintería de armar toledana por sus condiciones particulares, y porque hay indicios de que algunas armaduras podrían ser anteriores a las nazaríes de la Alhambra. La historia del oficio en el Toledo medieval merece ser revisada. Sirva este artículo para animar a la datación de armaduras con cronologías absolutas que permitan ordenar temporalmente los conceptos constructivos analizados y establecer las relaciones entre las diferentes zonas peninsulares y las distintas influencias capaces de generar la calidad y complejidad constructiva y decorativa de nuestra particular Carpintería de Armar.

\section{REFERENCIAS}

(1) Caviro, B. M. (1980). Mudéjar toledano: Palacios y conventos. S.l. : s.n. (Madrid: Vocal Art. Gráf.)

(2) Nuere, E., \& Colegio Oficial de Aparejadores y Arquitectos Técnicos de Toledo. (1992). Artesonados de Toledo. Toledo: Colegio Oficial de Aparejadores y Arquitectos Técnicos de Toledo.

(3) Castilla-La Mancha. (1991b). Arquitecturas de Toledo. Toledo: Junta de Comunidades de Castilla-La Mancha, Servicio de Publicaciones.

(4) Nuere, E., Universidad de Alcalá de Henares, \& Instituto Español de Arquitectura. (200o). La carpintería de armar española. Madrid: Munilla-Lería.

(5) Prieto y Vives, V. (1977). El arte de la lacería. Madrid: Madrid Colegio Oficial de Ingenieros de Caminos, Canales y Puertos.

(6) Fernández Cabo, M. (1991). Armaduras de cubierta en la Región Leonesa: Bases documentales y criterios para el análisis, clasificación y evolución de las tipologías estructurales de la cubierta en la Región Leonesa (tesis doctoral). Madrid: E.T.S. Arquitectura, Universidad Politécnica de Madrid.

(7) Carrasson, A. (14-16 septiembre 2011). Aspectos técnicos de la techumbre de la Catedral de Teruel, 103-116. En Actas XII Simposio Internacional de Mudejarismo, Teruel.

(8) Izquierdo Benito, R. (2012). Las iglesias de Toledo en la Edad Media. Evidencias arqueológicas. Discurso de apertura del Curso 2012-2013, Real Academia de Toledo.

(9) Nuere, E., \& San Miguel, A. d., Fray. (1990). La carpintería de lazo: Lectura dibujada del manuscrito de Fray Andrés de San Miguel, p. 96. Málaga: Colegio Oficial de Arquitectos de Málaga. 
(10) Mingo García, J. de (2014). Las techumbres de la Iglesia del Hospital de San Salvador en Buitrago de Lozoya. Boletín de la Real Academia de Bellas Artes de San Fernando, 116: 23-36.

(11) Nuere, E., \& López de Arenas, D. (2001). Nuevo tratado de la carpintería de lo blanco y la verdadera historia de Enrique Garavato carpintero de lo blanco y maestro del oficio. Madrid: Munilla-Lería. 